\title{
Auditory Evoked Potentials with Different Speech Stimuli: a Comparison and Standardization of Values
}

\author{
Dayane Domeneghini Didoné ${ }^{1}$ Sheila Jacques Oppitz ${ }^{1}$ Jordana Folgearini ${ }^{1} \quad$ Eliara Pinto Vieira Biaggio ${ }^{1}$ \\ Michele Vargas Garcia ${ }^{1}$ \\ ${ }^{1}$ Department of Phonoaudiology, Universidade Federal de Santa \\ Maria, Santa Maria, Brazil \\ Int Arch Otorhinolaryngol 2016;20:99-104.

\begin{abstract}
Address for correspondence Dayane Domeneghini Didoné, MSc, Universidade Federal de Santa Maria - Fonoaudiologia, Avenida Roraima, $n^{\circ} 1000$, Bairro Camobi, Rua João Goulart, Santa Maria RS 97105-220, Brazil (e-mail: dayanedidone@yahoo.com.br).
\end{abstract}

\begin{abstract}
Keywords

- evoked potentials

- auditory

- speech perception

- electrophysiology

Introduction Long Latency Auditory Evoked Potentials (LLAEP) with speech sounds has been the subject of research, as these stimuli would be ideal to check individuals detection and discrimination.

Objective The objective of this study is to compare and describe the values of latency and amplitude of cortical potentials for speech stimuli in adults with normal hearing.

Methods The sample population included 30 normal hearing individuals aged between 18 and 32 years old with ontological disease and auditory processing. All participants underwent LLAEP search using pairs of speech stimuli (/ba/ x/ga/, /ba/ x $/ \mathrm{da} /$, and $/ \mathrm{ba} / \mathrm{x} / \mathrm{di} /$. The authors studied the LLAEP using binaural stimuli at an intensity of $75 \mathrm{dBNPS}$. In total, they used 300 stimuli were used ( $\sim 60$ rare and 240 frequent) to obtain the LLAEP. Individuals received guidance to count the rare stimuli. The authors analyzed latencies of potential P1, N1, P2, N2, and P300, as well as the ampleness of P300.

Results The mean age of the group was approximately 23 years. The averages of cortical potentials vary according to different speech stimuli. The N2 latency was greater for /ba/ x / di/ and P300 latency was greater for /ba/ x/ga/. Considering the overall average amplitude, it ranged from 5.35 and $7.35 \mathrm{uV}$ for different speech stimuli. Conclusion It was possible to obtain the values of latency and amplitude for different speech stimuli. Furthermore, the N2 component showed higher latency with the / ba / $\mathrm{x}$ / di / stimulus and P300 for /ba/ $\mathrm{x}$ / ga /.
\end{abstract}

\section{Introduction}

Cortical Auditory Evoked Potentials (CAEPs) have gradually entered clinical practice, being useful to support diagnoses of central auditory disorders. Furthermore, this assessment can reflect neuroeletrical activity of the auditory pathways.

CAEPs can be elicited by both verbal and non-verbal stimuli, ${ }^{1,2}$ which reflect the neuroelectrical activity of the auditory pathway in the regions of the thalamus and auditory cortex. ${ }^{3}$ Several studies have aimed at analyzing electrophysiological assessment with speech stimuli, including the verification of peripheral auditory structures, as in the Auditory Brainstem Response (ABR). ${ }^{4-6}$

Some researchers suggest that verbal stimuli are ideal for studying the neural basis of speech detection and discrimination. ${ }^{1,2}$ Additionally, these types of stimuli can contribute received

May 11, 2015

accepted

July 26, 2015

published online

February 15, 2016
DOI http://dx.doi.org/

10.1055/s-0035-1566133. ISSN 1809-9777.
Copyright $\odot 2016$ by Thieme Publicações License terms Ltda, Rio de Janeiro, Brazil 
to the assessment of complex signals in the auditory cortex. Recent studies support the use of complex signals for the evaluation of retrocochlear pathologies, central auditory disorders, and verification of hearing aids. ${ }^{7,8}$

McPherson ${ }^{9}$ and McGee ${ }^{10}$ published normative values for the CAEPs with tone burst stimuli. Other studies have also reported normative values for the tonal stimuli. ${ }^{11-13}$ On the other hand, literature shows different values for verbal stimuli. ${ }^{14,15}$ Authors describe the latency and amplitude of P300 as sensitive to the task demand, and higher latency and lower amplitude for speech stimuli. ${ }^{16,17}$

Although the literature describes differences between tone burst and speech stimuli, the protocols for verbal stimuli vary in their application, the stimuli used, and the values of latency and amplitude. Thus, further studies should establish rules and criteria so that this procedure can be effectively applied in clinical practice.

The aim of this study is to compare and describe the values of latency and amplitude of CAEPs for speech stimuli in normal hearing adults, in order that the results may serve as a reference for clinical and research in audiology and other areas.

\section{Methods}

The Research Ethics Committee of the University where this study was conducted granted its approval for the study.

Participants who agreed to the research signed the term of responsibility. They received information on all procedures from this research.

All participants were aged between 18 and 32, male and female, with normal hearing, free from ear's disease or auditory process disorders, and without continuous use of medication. They needed to be able to understand all procedures.

The authors excluded from this study individuals with hearing loss and auditory processing disorder, guiding them to specific assessments.

All subjects underwent evaluation through anamnesis, visual inspection of the external acoustic meatus, audiometry, middle ear assessment, and long latency auditory evoked potentials with different verbal stimuli.

The anamnesis provided information on patientś audiological evolution and auditory processing disorder.

The patients were subject to a visual inspection of the external auditory meatus using a KlinicWelch-Allyn clinical otoscope (KlinicWelch-Allyn, NY, USA) to discard any pathological changes that might have influenced audiometric thresholds.

The authors performed audiometry in an acoustically treated place using the audiometer Itera II Madsen (Otometrics, Denmark). They tested the frequencies 250, 500, 1000, 2000, $3000,4000,6000$, and $8000 \mathrm{~Hz}$, using the descending-ascending technique. The study considered as normal hearing individuals those with three-tone average $(500,1000$, and $2000 \mathrm{~Hz}$ ) less than or equal to $25 \mathrm{~dB}$ HL (decibel hearing level). ${ }^{18}$

The acoustic impedance measurements were performed by AT235 Interacoustics (Middelfart, Denmark). All participants were submitted to tympanometric curve and acoustic reflexes. The authors analyzed reflexes in the frequency range from 500 to $4000 \mathrm{~Hz}$, bilaterally in the contralateral mode.
The study included only individuals with type " $\mathrm{A}$ " tympanogram presenting acoustic reflexes. ${ }^{19}$

The CAEPs were performed with the Intelligent Hearing Systems (IHS), SmartEP module. This equipment contains two response channels. The skin on all subjects was cleaned with an abrasive paste. The electrodes were placed in the following positions: A1 (left mastoid) and A2 (right mastoid), $\mathrm{Cz}$ (vertex), the ground electrode (Fpz) on the forehead. The impedance value for all electrodes was less than or equal to 3 kohms.

The patient received instructions to pay attention to different stimuli (rare stimuli) that appeared within a series of equal stimuli (frequent stimuli). The percentage of rare stimuli presented was $20 \%$, while for frequent stimuli was $80 \%$.

The speech tokens stimuli used were the consonant-vowel /ba/ as frequent stimuli in all sequences, compared with different rare stimuli, as /ga/, /da/, and /di/. Therefore, a sequence of different deviant stimuli were tested (/ba/ x /ga/, /ba/ x /da/, /ba/ $\mathrm{x} / \mathrm{di} /$ ). All speech token stimuli were presented in both ears at an intensity of $75 \mathrm{~dB}$ HL. In total, 300 stimuli were used (60 rare and 240 frequent) to obtain the CAEPs.

The assessment began with /ba/ x/ga/, followed by /ba/ $\mathrm{x}$ /di/ and /ba/ $x$ /da/. Prior to obtaining the results, all participants received training to listen to the verbal stimuli to become familiar with them. The patients had to report to the evaluator the number of rare stimuli. The evaluator compared the response with the number of rare stimuli effectively presented by the equipment. For the answer to be considered correct there was a margin of error adopted of up to five stimuli that differed from the exact amount presented by the equipment.

The authors obtained latency values for CAEPs to identify the waves in the greater range and deflection peaks. They did not replicate results as this could tire the individual and jeopardize the outcome of the assessment. The amplitude was measured only for the P300 component, calculated from the baseline to the peak of the component.

The authors described and analyzed the results statistically using the Post Hoc Bonferroni test. They compared latencies of P1, N1, P2, N2, and P300 and the amplitude of P300 between the speech sounds. - Table 1 describes the parameters used in this study.

\section{Results}

In total, the researchers evaluated 30 subjects, of which 15 (50\%) were male and 15 (50\%) female. Their average age was $23.3( \pm 3.5)$ years.

Although stimulation has been binaural, the two-channel equipment allowed responses for the right and left ears. The results were statistically analyzed using the Bonferroni post hoc test and no statistically significant difference were found between ears, both for latency and amplitude. Therefore, to facilitate the analysis of this study, the authors grouped the results of the right and left ears.

- Table 2 shows the percentage of presence of CAEPs for different stimuli. The other results were obtained from all CAEPs considered present. 
Table 1 Parameters used in this research of CAEPs with speech stimuli

\begin{tabular}{|l|l|}
\hline Equipment & Intelligent Hearing System \\
\hline Module & SmartEP \\
\hline Electrodes & A1, A2, Fpz and Cz \\
\hline Impedance of electrodes & Less or equal to 3 kohms \\
\hline Type of stimulation & Binaural \\
\hline Number of stimulus & 300 (80\% frequent and 20\% rare) \\
\hline Channels & AB \\
\hline Rate & 0.8 pps \\
\hline Time & 2.0 milliseconds \\
\hline Phase & Alternating pattern \\
\hline Speech tokens & $\begin{array}{l}\text { /ba/ (frequent) /ga/ (rare) } \\
\text { /ba/ (frequent) /di/ (rare) } \\
\text { /ba/ (frequent) /da/ (rare) }\end{array}$ \\
\hline Presentation of stimulus & Oddball paradigm \\
\hline Time of stimulus & 50.000 Hs \\
\hline Rise and decay time & $20 \%$ \\
\hline Envelope's stimulus & Trapezoidal \\
\hline Individual state & Alert \\
\hline
\end{tabular}

Abbreviations: Kohms, kiloohms; ms, milliseconds; pps, pulses per second; $\mu$ s, microseconds.

- Table 3 describes the latency values of CAEPs for all speech tokens. There was no statistically significant difference between latencies of P1, N1, and P2. However, the latency for N2 was greater for /ba/ x /di/ stimuli, and this difference was statistically significant. For the P300 component, there was statistically significant differences between speech tokens, being higher for the /ba/ x /ga/ stimulus.

- Table 4 describes the P300 amplitude values for different speech stimuli. There was no statistically significant difference between stimuli.

- Table 5 shows the maximum and minimum descriptive values for the all variables studied.

\section{Discussion}

Electrophysiological studies using complex stimuli have been increasingly prominent in national and international literature. In general, more complex speech stimuli evoke greater latencies and lower amplitudes of CAEPs. In addition, natural speech stimuli evoke lower latencies compared with synthetic stimuli generated by the equipment. ${ }^{20}$

In this study, the percentage of presence for N1-P2 was greater than other CAEPs. However, P1 and N2 were mainly affected by the speech tokens characteristics. This can be explained by the fact that the N1-P2 complex is the most visible exogenous potentials, which makes it less variable in relation to stimuli. ${ }^{21,22}$ Our results are consistent with another study, ${ }^{8}$ in which the percentage of presence was lowest for N2. Research studies ${ }^{23}$ report that the cortical components are influenced by cognitive experiences of the individual throughout his life. Thus, better individual experiences with hearing, cognition, and music produce the best results in the CAEPs, meaning greater amplitudes and lower latencies.

In this study, the authors correlate their values of latency and amplitude with other similar studies considering the standard deviation. When values from other studies fall within up to two standard deviations from those presented herein, they are considered concordant.

In comparing the latencies of CAEPs for different stimuli, only the N2 component presents a significant difference, being higher for /ba/ x / di/. This component (N2) suffers maturational influences, mainly from 5 to 10 years, reducing latency and amplitude. ${ }^{24}$ The results from this research agrees with another study, ${ }^{25}$ in which the $\mathrm{N} 2$ component also suffered influence according to the type of stimuli presented. The authors emphasize that N2 is associated with attention to rare stimuli, and depends on the complexity of the stimuli; thus, the higher the complexity, the higher the latency.

For the P300, there was also difference between stimuli, with greater latency found in the /ba/ $\mathrm{x} / \mathrm{ga} / \mathrm{stimulus.}$ Another study ${ }^{25}$ reported similar results in in which the latency of P300 was higher for stimuli of greater complexity. Researchers $^{26}$ also reported increased latencies in more difficult tasks. In this study, the authors did not investigate

Table 2 Percentage of presence of cortical auditory evoked potentials with different speech stimulus

\begin{tabular}{|c|c|c|c|c|c|c|}
\hline \multicolumn{7}{|c|}{ Speech tokens } \\
\hline & \multicolumn{2}{|c|}{ /ba/ x /ga/ } & \multicolumn{2}{|c|}{ /ba/ x /da/ } & \multicolumn{2}{|c|}{ /ba/ x /di/ } \\
\hline & $\mathrm{N}$ & $\%$ & $\mathrm{~N}$ & $\%$ & $\mathrm{~N}$ & $\%$ \\
\hline P1 & 26 & $86.7 \%$ & 27 & $90 \%$ & 25 & $83.3 \%$ \\
\hline N1 & 30 & $100 \%$ & 30 & $100 \%$ & 30 & $100 \%$ \\
\hline P2 & 30 & $100 \%$ & 30 & $100 \%$ & 30 & $100 \%$ \\
\hline N2 & 23 & $76.7 \%$ & 16 & $53.3 \%$ & 14 & $46.7 \%$ \\
\hline P300 & 26 & $86.7 \%$ & 28 & $93.3 \%$ & 25 & $83.3 \%$ \\
\hline
\end{tabular}

Abbreviations: \%, percentage of presence; $\mathrm{N}$, number of subjects. 
Table 3 Average and standard deviation of latencies and amplitude for different speech stimulus

\begin{tabular}{|c|c|c|c|c|c|c|c|}
\hline \multicolumn{8}{|c|}{ Speech tokens } \\
\hline & \multicolumn{2}{|l|}{ /ba/ x /ga/ } & \multicolumn{2}{|l|}{$|\mathrm{ba} / \times| \mathrm{da} \mid$} & \multicolumn{3}{|l|}{ /ba/ x /di/ } \\
\hline & Average (ms) & SD (ms) & Average (ms) & SD (ms) & Average (ms) & SD (ms) & ${ }^{*}$ p-value \\
\hline P1 & 62.4 & 9.5 & 60.1 & 7.55 & 66.35 & 17.9 & 0.393 \\
\hline N1 & 103.55 & 10.45 & 103.5 & 11.4 & 108.55 & 18.05 & 0.038 \\
\hline P2 & 175.05 & 18.45 & 175.6 & 22.45 & 184.9 & 25.15 & 0.026 \\
\hline N2 & 250.5 & 33.3 & 234.8 & 41.05 & 256.5 & 35.45 & 0.006 \\
\hline P300 & 342.05 & 45.35 & 302.45 & 46.9 & 327.05 & 61.3 & 0.005 \\
\hline
\end{tabular}

Abbreviations: ms, millisecond; SD, standard deviation.

${ }^{*}$ Post Hoc Bonferroni test.

Table 4 Average and standard deviation of P300 amplitude

\begin{tabular}{|c|c|c|c|c|c|c|c|}
\hline \multicolumn{8}{|c|}{ Speech tokens } \\
\hline & \multicolumn{2}{|l|}{ /ba/ x /gal } & \multicolumn{2}{|l|}{ |ba/ x/da } & \multicolumn{3}{|l|}{ /ba/ x /di } \\
\hline & Average (uv) & SD (uv) & Average (uv) & SD (uv) & Average (uv) & SD (uv) & ${ }^{*} \mathrm{p}$-value \\
\hline P300 & 6.4 & 2.15 & 7.35 & 5.35 & 6.5 & 2.65 & 0.208 \\
\hline
\end{tabular}

Abbreviations: SD, standard deviation; uV, microvolt.

*Post Hoc Bonferroni.

Table 5 Maximum and minimum values of the variables

\begin{tabular}{|c|c|c|c|c|c|c|}
\hline \multicolumn{7}{|l|}{ Speech tokens } \\
\hline & \multicolumn{2}{|l|}{ /ba/ x /ga/ } & \multicolumn{2}{|l|}{ |ba $/ x /$ da $\mid$} & \multicolumn{2}{|l|}{ /ba/ x /di $/$} \\
\hline & $\operatorname{Max}(\mathrm{ms})$ & $\operatorname{Min}(\mathrm{ms})$ & $\operatorname{Max}(\mathrm{ms})$ & $\operatorname{Min}(\mathrm{ms})$ & $\operatorname{Max}(\mathrm{ms})$ & $\operatorname{Min}(\mathrm{ms})$ \\
\hline P1 & 86 & 56 & 64 & 50 & 76 & 50 \\
\hline N1 & 122 & 84 & 132 & 90 & 134 & 82 \\
\hline $\mathrm{P} 2$ & 224 & 136 & 220 & 150 & 226 & 142 \\
\hline $\mathrm{N} 2$ & 286 & 166 & 288 & 178 & 302 & 180 \\
\hline P300 & 430 & 210 & 430 & 220 & 446 & 236 \\
\hline Ampl P300(uV) & 12 & 3.00 & 24.59 & 3.04 & 23.4 & 3.01 \\
\hline
\end{tabular}

Abbreviations: Max, maximum; Min, minimum; ms, millisecond; uV, microvolt.

the spectral complexity of stimuli; nonetheless, participants informally reported that the $/ \mathrm{ba} / \mathrm{x} / \mathrm{ga} /$ stimuli was the most difficult to identify. This may justify the results for P300.

The P300 amplitude depends on the tasks performed by the individual. The amplitude increases in accordance with attentional parameters and receives influence from cognitive disorders. ${ }^{27}$ In our study, the overall average amplitude of P300 ranged between 5.35 and $7.35 \mathrm{uV}$ (microvolts) for all the different stimuli, and the overall average was $6.75 \mathrm{uV}$. Considering up to two standard deviations (mean of all stimuli DP), our results are in agreement with another study ${ }^{17}$ in which the authors found mean values of 6.61
$\mathrm{uV}$ for P300. There is a variation in the literature of 1.7 to $20 \mathrm{uV}$, and many authors do not use these values in interpreting the results because of the wide range of values described. ${ }^{28}$ In our study, we also found ample variation for the P300's amplitude in the values between all the different speech tokens.

Regarding the descriptive measures, some researches ${ }^{17}$ propose that the latency of P300 for verbal stimuli must be between $289.57 \mathrm{~ms}$ and $408.33 \mathrm{~ms}$. In our study, various speech stimuli were used. Nevertheless, considering the average values between the three stimuli $(323.85 \mathrm{~ms})$, our results agree with that proposed by the aforementioned authors. 
Table 6 Range latency and amplitude of cortical potentials obtained in this study

\begin{tabular}{|c|c|c|c|}
\hline \multicolumn{4}{|l|}{ Speech tokens } \\
\hline & /ba/ x /ga/ & /ba/ x/da & /ba/ x /di/ \\
\hline $\mathrm{P} 1$ (ms) (2SD) & $43.4-81.4$ & $45-75.2$ & $30.5-102.1$ \\
\hline $\mathrm{N} 1$ (ms) (2SD) & $82.6-124.4$ & $80.7-126.3$ & $72.4-144.6$ \\
\hline $\mathrm{P} 2$ (ms) (2SD) & $138.1-211.9$ & $130.7-220.5$ & $134.6-235.2$ \\
\hline $\mathrm{N} 2$ (ms) (2SD) & $183.9-317.1$ & $152.7-316.9$ & $185.6-327.4$ \\
\hline P300 (ms) (2SD) & $251.3-432.7$ & $208.6-396.2$ & $204.4-449.6$ \\
\hline Amplitude of P300 (uV) (1SD) & $4.25-8.55$ & $2.0-12.7$ & $3.85-9.15$ \\
\hline
\end{tabular}

Abbreviations: SD, standard deviation; uV, microvolt.

We found no published papers reporting the amplitude and latency of CAEPs for specific stimuli speech, such as /ba/ $\mathrm{x} / \mathrm{ga} /, \mathrm{ba} / \mathrm{x} / \mathrm{da} /$, and /ba/ x /di/. Thus, the table below (-Table 6) suggests values, norms, and comparisons based on the average, considering up to two standard deviations for latency and up to one standard deviation for the amplitude of P300. We determined only one standard deviation for the amplitude due to the wide variation of results. These results are useful for future studies that use the same speech tokens.

The description and comparison of these values is important for clinical use. The audiologist must understand the changes in cortical potentials for different stimuli. The stimuli selected may compromise the results of the evaluations due to their complexity. Therefore, the use of speech tokens is recommended as long as it is possible to know the different results in our clinical practice.

\section{Conclusion}

This study demonstrates that the protocol for speech stimuli described produces similar results from different stimuli, albeit the latency of $\mathrm{N} 2$ was higher for /ba/ x / di/, while the P300 amplitude was greater for /ga/ x /ba/. Moreover, the description of amplitude and latency values for different speech stimuli provide useful material for future studies.

\section{References}

1 Kraus N, Nicol T. Aggregate neural responses to speech sounds in the central auditory system. Speech Commun 2003;41:35-47

2 Korczak PA, Kurtzberg D, Stapells DR. Effects of sensorineural hearing loss and personal hearing AIDS on cortical event-related potential and behavioral measures of speech-sound processing. Ear Hear 2005;26(2):165-185

3 Soares AJC, Sanches SGG, Neves-Lobo IF, Carvalho RMM, Matas CG, Cárnio MS. Potenciais evocados auditivos de longa latência e processamento auditivo central em crianças com alterações de leitura e escrita: dados preliminares. Int Arch Otorhinolaryngol 2011;15(4):486-491

4 Banai K, Hornickel J, Skoe E, Nicol T, Zecker S, Kraus N. Reading and subcortical auditory function. Cereb Cortex 2009;19(11):2699-2707
5 Skoe E, Kraus N. Auditory brain stem response to complex sounds: a tutorial. Ear Hear 2010;31(3):302-324

6 Malayeri S, Lotfi Y, Moossavi SA, Rostami R, Faghihzadeh S. Brainstem response to speech and non-speech stimuli in children with learning problems. Hear Res 2014;313:75-82

7 Sharma A, Nash AA, Dorman M. Cortical development, plasticity and re-organization in children with cochlear implants. J Commun Disord 2009;42(4):272-279

8 Alvarenga KF, Amorim RB, Agostinho-Pesse RS, Costa OA, Nascimento LT, Bevilacqua MC. Speech perception and cortical auditory evoked potentials in cochlear implant users with auditory neuropathy spectrum disorders. Int J Pediatr Otorhinolaryngol 2012; 76(9):1332-1338

9 Mcpherson DL. Late potentials of auditory system (evoked potentials). San Diego: Singular Publishing Group; 1996

10 Kraus N, Mc Gee T. Potenciais auditivos evocados de longa latência. In: Katz J, editor. Handbook of Clinical Audiology. Baltimore: Williams and Wilkins; 1994:406-23

11 Crippa BL, Aita ADC, Ferreira MIDC. Padronização das respostas eletrofisiológicas para o P300 em adultos normouvintes. Distúrb Comum 2011;23:325-333

12 Duarte JL, Alvarenga KF, Banhara MR, Mello ADP, Sás RM, Costa Filho OA. Potencial evocado auditivo de longa latência-P300 em indivíduos normais: valor do registro simultâneo em Fz e Cz. Braz J Otorhinolaryngol 2009;75:231-236

13 Machado CSS, Carvalho ACO, Silva PLG. Caracterização da normalidade do P300 em adultos jovens. Rev Soc Bras Fonoaudiol 2009; 14:83-90

14 Uppenkamp S, Johnsrude IS, Norris D, Marslen-Wilson W, Patterson RD. Locating the initial stages of speech-sound processing in human temporal cortex. Neuroimage 2006; 31(3):1284-1296

15 Samson F, Zeffiro TA, Toussaint A, Belin P. Stimulus complexity and categorical effects in human auditory cortex: an activation likelihood estimation meta-analysis. Front Psychol 2010;1: 241

16 Martin BA, Tremblay KL, Korczak P. Speech evoked potentials: from the laboratory to the clinic. Ear Hear 2008;29(3): 285-313

17 Massa CGP, Rabelo CM, Matas CG, Schochat E, Samelli AG. P300 with verbal and nonverbal stimuli in normal hearing adults. Braz J Otorhinolaryngol 2011;77(6):686-690

18 Lloyd II Kaplan. 1978 apud Momenshon-Santos TM; Russo ICP; Brunetto-Borgianni LM. Interpretação dos resultados da avaliação audiológica. In: Momenshon-Santos TM, Russo ICP. Prática da audiologia clínica. 6ed. São Paulo: Cortez; 2007

19 Jerger J. Clinical experience with impedance audiometry. Arch Otolaryngol 1970;92(4):311-324 
20 Swink S, Stuart A. Auditory long latency responses to tonal and speech stimuli. J Speech Lang Hear Res 2012;55(2): 447-459

21 Albrecht R, Suchodoletz W, Uwer R. The development of auditory evoked dipole source activity from childhood to adulthood. Clin Neurophysiol 2000;111(12): 2268-2276

22 Martin B, Tremblay K, Stapells D. Principles and applications of cortical auditory evoked potentials. In: Eggermont J, Don M. Auditory evoked potentials: basic principles and clinical application. Baltimore2007:482-507

23 Polat Z, Ataş A. The investigation of cortical auditory evoked potentials responses in young adults having musical education. Balkan Med J 2014;31(4):328-334
24 Ponton CW, Eggermont JJ, Kwong B, Don M. Maturation of human central auditory system activity: evidence from multi-channel evoked potentials. Clin Neurophysiol 2000;111(2):220-236

25 Alvarenga KF, Vicente LC, Lopes RAF, et al. The influence of speech stimuli contrast in cortical auditory evoked potentials. Braz J Otorhinolaryngol 2013;79(3):336-341

26 Geal-Dor M, Kamenir Y, Babkoff H. Event related potentials (ERPs) and behavioral responses: comparison of tonal stimuli to speech stimuli in phonological and semantic tasks. J Basic Clin Physiol Pharmacol 2005;16(2-3):139-155

27 Polich J, Herbst KL. P300 as a clinical assay: rationale, evaluation, and findings. Int J Psychophysiol 2000;38(1):3-19

28 Reis ACMB, Iório MCM. P300 em sujeitos com perda auditiva. Prófono Rev Atual Cient 2007;19:113-122 